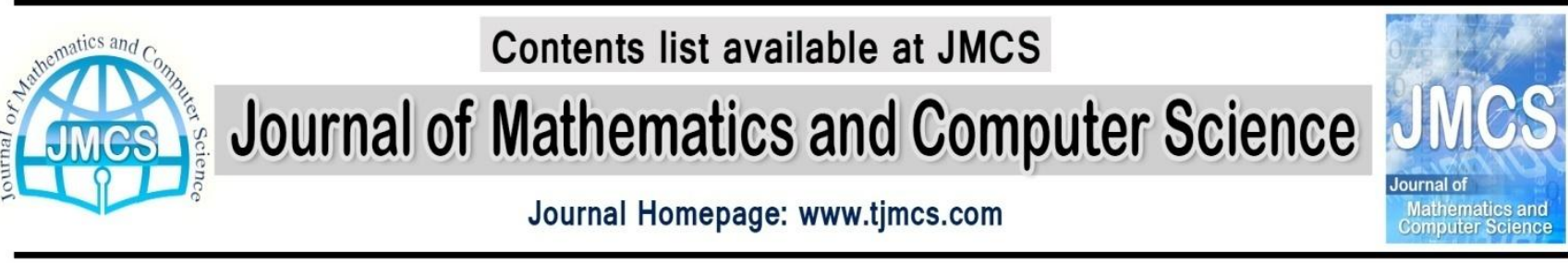

\section{Application of reduced differential transformation method for solving Fourth-Order Parabolic Partial Differential Equations}

\author{
Birol ỉiş \\ Department of Main Sciences, Turkish Air Force Academy, 34149 Yeşilyurt, Istanbul, Turkey \\ bibis@hho.edu.tr
}

\author{
Article history: \\ Received July 2014 \\ Accepted August 2014 \\ Available online August 2014
}

\begin{abstract}
The purpose of this paper is to obtain the approximate solution of fourth-order parabolic partial differential equations by the reduced differential transform method (RDTM).This method provides the solution in the form of a convergent series with easily calculable terms. Comparing RDTM with some other methods in the literature shows present approach is very simple, effective, powerful and can be easily applied to other linear or nonlinear PDEs in science and engineering.
\end{abstract}

Keywords: Reduced differential transform method (RDTM), differential transform method (DTM) fourthorder parabolic partial differential equations, initial value problems.

\section{Introduction}

There are many problems arising in science and engineering are modeled using linear or nonlinear partial differential equations (PDEs). Boundary and initial value problems in PDEs occur in fluid mechanics, mathematical physics, astrophysics, biology, materials science, electromagnetism, image processing, computer graphics, etc. PDEs are categorized into different types, including elliptic, parabolic, and hyperbolic PDEs. In this article we concentrate our discussion on fourth-order parabolic PDEs. These PDEs describe various physical phenomenon including deformation of beams, viscoelastic and inelastic flows, transverse vibrations of a homogeneous beam, plate deflection theory, engineering and applied sciences [1-12]. In recent years, various methods have been proposed for solving the fourth-order parabolic PDEs, such that adomian decomposition method (ADM) [13,14], variational iteration method (VIM) [15,16], B-spline methods [17-19], homotopy perturbation method (HPM) [20] and homotopy analysis method (HAM) [21].

In this paper, we applied the RDTM, which is the modified version of differential transform method (DTM), to fourth-order parabolic PDEs. RDTM doesn't require any discretization or linearization and it reduces significantly the computational work. Also, it provides an analytical approximation, in many cases an exact solution, in a rapidly convergent sequence with elegantly computed terms. computational work. 


\section{Reduced differential transform method (RDTM)}

In this section, some basic definitions and properties for RDTM, which could be found in [22-26], have been reviewed.

Definition 2.1. Consider a function of $\mathrm{n}+1$ variables $u(\tilde{x}, t)=u\left(x_{1}, x_{2}, \ldots x_{n}, t\right)$ where $\tilde{x} \in R^{n}$, $\tilde{x}=\left(x_{1}, x_{2}, \ldots, x_{n}\right)$. The reduced differential transform of $u(\tilde{x}, t)$ with respect to $t$ is defined by

$$
U_{k}(\tilde{x})=\frac{1}{k !}\left[\frac{\partial^{k}}{\partial t^{k}} u(\tilde{x}, t)\right]_{t=0} \quad, k \in N
$$

In Eq.(1) $U_{k}(\tilde{x})$ is the transformed function and $u(\tilde{x}, t)$ is the original function.

Definition 2.2. The reduced differential inverse transform of $U_{k}(\tilde{x})$ is defined as follows:

$$
u(\tilde{x}, t)=\sum_{k=0}^{\infty} U_{k}(\tilde{x}) \cdot t^{k}
$$

From Eq.(1) and Eq.(2) , we get

$$
u(\tilde{x}, t)=\sum_{k=0}^{\infty} \frac{t^{k}}{k !}\left[\frac{\partial^{k}}{\partial t^{k}} u(\tilde{x}, t)\right]_{t=0}
$$

Notice that the RDTM is close to the one dimensional DTM because the RDTM is considered as the standard DTM of $u(\tilde{x}, t)$ with respect to the variable t. However, the corresponding recursive algebraic equation is the function of the variable $\tilde{x}=\left(x_{1}, x_{2}, \ldots, x_{n}\right)$.

The following theorems that can be deduced from Eqs.(1)-(3) are given below:

Theorem 1. If $f(\tilde{x}, t)=\alpha g(\tilde{x}, t) \pm \beta h(\tilde{x}, t)$, then $F_{k}(\tilde{x})=\alpha G_{k}(\tilde{x}) \pm \beta H_{k}(\tilde{x})$, where $\alpha$ and $\beta$ are constant.

Theorem 2. If $f(\tilde{x}, t)=x_{1}^{m_{1}} x_{2}^{m_{2}} \cdots x_{n}^{m_{n}} t^{n}$, then $F_{k}(\tilde{x})=x_{1}^{m_{1}} x_{2}^{m_{2}} \cdots x_{n}^{m_{n}} \delta(k-n)$ where $\delta(k)=\left\{\begin{array}{l}1, k=0 \\ 0, k \neq 0\end{array}\right.$.

Theorem 3. If $f(\tilde{x}, t)=g(\tilde{x}, t) \cdot h(\tilde{x}, t)$, then $F_{k}(\tilde{x})=\sum_{r=0}^{k} G_{r}(\tilde{x}) \cdot H_{k-r}(\tilde{x})$

Theorem 4. If $f(\tilde{x}, t)=x_{1}^{m_{1}} \cdot x_{2}^{m_{2}} \cdots x_{n}^{m_{n}} \cdot t^{n} \cdot g(\tilde{x}, t)$, then $F_{k}(\tilde{x})=x_{1}^{m_{1}} \cdot x_{2}^{m_{2}} \cdots x_{n}^{m_{n}} \cdot G_{k-n}(\tilde{x})$

Theorem 5. If $f(\tilde{x}, t)=\frac{\partial^{n}}{\partial t^{n}} g(\tilde{x}, t)$, then $F_{k}(\tilde{x})=(k+1)(k+2) \cdots(k+n) G_{k+n}(\tilde{x})$

Theorem 6. If $f(\tilde{x}, t)=\frac{\partial^{n}}{\partial x_{i}^{n}} g(\tilde{x}, t)$, then $F_{k}(\tilde{x})=\frac{\partial^{n}}{\partial x_{i}^{n}} G_{k}(\tilde{x}), i=1,2, \ldots, n$

Theorem 7. If $f(\tilde{x}, t)=\sin \left(\sum_{i=1}^{n} \alpha_{i} x_{i}+\beta t\right)$, then $F_{k}(\tilde{x})=\left(\frac{\beta^{k}}{k !}\right) \cdot \sin \left(\frac{k \pi}{2}+\sum_{i=1}^{n} \alpha_{i} x_{i}\right)$

Theorem 8. If $f(\tilde{x}, t)=\cos \left(\sum_{i=1}^{n} \alpha_{i} x_{i}+\beta t\right)$, then $F_{k}(\tilde{x})=\left(\frac{\beta^{k}}{k !}\right) \cdot \cos \left(\frac{k \pi}{2}+\sum_{i=1}^{n} \alpha_{i} x_{i}\right)$ 


\section{Applications of RDTM to fourth-order parabolic PDEs}

In this section, in order to show the applicability and efficiency of the RDTM for solving the fourthorder parabolic PDEs, some illustrative examples are given.

Example 3.1 Firstly, consider the following one dimensional non-homogeneous fourth-order parabolic PDE [13].

$$
\frac{\partial^{2} u}{\partial t^{2}}+(1+x) \frac{\partial^{4} u}{\partial x^{4}}=\left(x^{4}+x^{3}-\frac{6}{7 !} x^{7}\right) \cos t, 0<x<1, t>0
$$

subject to the initial condition

$$
u(x, 0)=\frac{6}{7 !} x^{7}, \frac{\partial u}{\partial t}(x, 0)=0
$$

whose exact solution is

$$
u(x, t)=\frac{6}{7 !} x^{7} \cos t
$$

By applying the RDTM on Eq.(4), the following recursive equation is obtained:

$$
U_{k+2}(x)=\frac{-1}{(k+1)(k+2)}\left\{(1+x) \frac{\partial^{4}}{\partial x^{4}} U_{k}(x)+F_{k}(x)\right\}
$$

where $F_{k}(x)$ is the transformation of the function $f(x, t)=\left(x^{4}+x^{3}-\frac{6}{7 !} x^{7}\right)$ cost. From theorem 8 ,
the reduced differential transform $F_{k}(x)$ is,

$$
F_{k}(x)=\left(x^{4}+x^{3}-\frac{6}{7 !} x^{7}\right) \frac{1}{k !} \cos \left(\frac{k \pi}{2}\right)
$$

From Eq.(1), the initial conditions given in Eq. (5) can be transformed at $t=0$ as

$$
U_{0}(x)=\frac{6}{7 !} x^{7}, U_{1}(x)=0
$$

Substituting Eqs.(8)-(9) into Eq.(7) and by straightforward iterative steps, the following $U_{k}(x)$ (for $\mathrm{k}=0,1,2, \ldots, \mathrm{n})$ values are obtained.

$$
\begin{aligned}
& U_{0}(x)=\frac{6}{7 !} x^{7}, U_{1}(x)=0, U_{2}(x)=-\frac{6}{7 !} \cdot \frac{1}{2} x^{7}, U_{3}(x)=0, U_{4}(x)=\frac{6}{7 !} \cdot \frac{1}{24} x^{7} \\
& U_{5}(x)=0, U_{6}(x)=-\frac{6}{7 !} \cdot \frac{1}{6 !} x^{7}, U_{7}(x)=0, U_{8}(x)=\frac{6}{7 !} \cdot \frac{1}{8 !} x^{7}, \cdots
\end{aligned}
$$

Then, using the inverse transformation Eq.(2), the 8th order approximate solution as,

$$
\begin{aligned}
\tilde{u}(x, t)=\sum_{k=0}^{8} U_{k}(x) t^{k} & =\frac{6}{7 !} x^{7}-\frac{6}{7 !} \cdot \frac{1}{2 !} x^{7} t^{2}+\frac{6}{7 !} \cdot \frac{1}{4 !} x^{7} t^{4}-\frac{6}{7 !} \cdot \frac{1}{6 !} x^{7} t^{6}+\frac{6}{7 !} \cdot \frac{1}{8 !} x^{7} t^{8}+\cdots \\
& =\frac{6}{7 !} x^{7}\left(1-\frac{1}{2 !} t^{2}+\frac{1}{4 !} t^{4}-\frac{1}{6 !} t^{6}+\frac{1}{8 !} t^{8}+\cdots\right)
\end{aligned}
$$


which is the first eight terms of the poisson series of the exact solution Eq.(6).

Example 3.2 Now, Consider the following singular fourth-order parabolic PDE in two space variables [14]

$$
\frac{\partial^{2} u}{\partial t^{2}}+2\left(\frac{1}{x^{2}}+\frac{x^{4}}{6 !}\right) \frac{\partial^{4} u}{\partial x^{4}}+2\left(\frac{1}{y^{2}}+\frac{y^{4}}{6 !}\right) \frac{\partial^{4} u}{\partial y^{4}}=0, \frac{1}{2}<x, y<1, t>0
$$

subject to the initial condition

$$
u(x, y, 0)=0, \frac{\partial u}{\partial t}(x, y, 0)=2+\frac{x^{6}}{6 !}+\frac{y^{6}}{6 !}
$$

whose exact solution is

$$
u(x, y, t)=\left(2+\frac{x^{6}}{6 !}+\frac{y^{6}}{6 !}\right) \sin t
$$

Using above theorems, the transformed form of Eq. (11) is find as

$$
U_{k+2}(x, y)=\frac{-1}{(k+1)(k+2)}\left\{2\left(\frac{1}{x^{2}}+\frac{x^{4}}{6 !}\right) \frac{\partial^{4} U_{k}(x, y)}{\partial x^{4}}+2\left(\frac{1}{y^{2}}+\frac{y^{4}}{6 !}\right) \frac{\partial^{4} U_{k}(x, y)}{\partial y^{4}}\right\}
$$

From Eq.(1), the initial conditions given in Eq. (12) can be transformed at $t=0$ as

$$
U_{0}(x, y)=0, U_{1}(x, y)=2+\frac{x^{6}}{6 !}+\frac{y^{6}}{6 !}
$$

Substituting Eq.(15) into Eq.(14) and by straightforward iterative steps, the following $U_{k}(x)$ (for $\mathrm{k}=0,1,2, \ldots, \mathrm{n})$ values are obtained.

$$
\begin{aligned}
& U_{0}(x)=0, U_{1}(x)=2+\frac{x^{6}}{6 !}+\frac{y^{6}}{6 !}, U_{2}(x)=0, U_{3}(x)=-\frac{1}{3 !}\left(2+\frac{x^{6}}{6 !}+\frac{y^{6}}{6 !}\right), U_{4}(x)=0 \\
& U_{5}(x)=\frac{1}{5 !}\left(2+\frac{x^{6}}{6 !}+\frac{y^{6}}{6 !}\right), U_{6}(x)=0, U_{7}(x)=-\frac{1}{7 !}\left(2+\frac{x^{6}}{6 !}+\frac{y^{6}}{6 !}\right), U_{8}(x)=0, \cdots
\end{aligned}
$$

Then, using the inverse transformation Eq.(2), we get the 8th order approximate solution as,

$$
\begin{aligned}
\tilde{u}(x, t)=\sum_{k=0}^{n} U_{k}(x) t^{k} & =\left(2+\frac{x^{6}}{6 !}+\frac{y^{6}}{6 !}\right) t-\frac{1}{3 !}\left(2+\frac{x^{6}}{6 !}+\frac{y^{6}}{6 !}\right) t^{3}+\frac{1}{5 !}\left(2+\frac{x^{6}}{6 !}+\frac{y^{6}}{6 !}\right) t^{5} \\
& -\frac{1}{7 !}\left(2+\frac{x^{6}}{6 !}+\frac{y^{6}}{6 !}\right) t^{7} \\
& =\left(2+\frac{x^{6}}{6 !}+\frac{y^{6}}{6 !}\right)\left(t-\frac{1}{3 !} t^{3}+\frac{1}{5 !} t^{5}-\frac{1}{7 !} t^{7}\right)
\end{aligned}
$$

which is the first eight terms of the poisson series of the exact solution Eq.(13).

Example 3.3 Consider the following PDE in three space variables [14] 


$$
\frac{\partial^{2} u}{\partial t^{2}}+\left(\frac{y+z}{2 \cos x}-1\right) \frac{\partial^{4} u}{\partial x^{4}}+\left(\frac{z+x}{2 \cos y}-1\right) \frac{\partial^{4} u}{\partial y^{4}}+\left(\frac{x+y}{2 \cos z}-1\right) \frac{\partial^{4} u}{\partial z^{4}}=0,0<x, y, z<\pi / 3, t>0
$$

subject to the initial condition

$$
u(x, y, z, 0)=-\frac{\partial u}{\partial t}(x, y, z, 0)=(x+y+z)-(\cos x+\cos y+\cos z)
$$

whose exact solution is

$$
u(x, y, z, t)=(x+y+z-\cos x-\cos y-\cos z) e^{-t}
$$

Using the RDTM, , the transformed form of Eq. (17) can be viewed as the following recursive formula

$$
\begin{aligned}
U_{k+2}(x, y, z) & =\frac{-1}{(k+1)(k+2)}\left\{\left(\frac{y+z}{2 \cos x}-1\right) \frac{\partial^{4} U_{k}(x, y, z)}{\partial x^{4}}+\left(\frac{z+x}{2 \cos y}-1\right) \frac{\partial^{4} U_{k}(x, y, z)}{\partial y^{4}}\right. \\
\left.+\left(\frac{x+y}{2 \cos z}-1\right) \frac{\partial^{4} U_{k}(x, y, z)}{\partial z^{4}}\right\} &
\end{aligned}
$$

From Eq.(1), the initial conditions given in Eq.(18) can be transformed at $t=0$ as

$$
U_{0}(x, y, z)=-U_{1}(x, y, z)=(x+y+z)-(\cos x+\cos y+\cos z)
$$

Substituting Eq.(21) into Eq.(20) and by straightforward iterative steps, we get the following $U_{k}(x)$ (for $\mathrm{k}=0,1,2, \ldots, \mathrm{n}$ ) values.

$$
\begin{aligned}
& U_{0}(x)=(x+y+z-\cos x-\cos y-\cos z), \quad U_{1}(x)=-(x+y+z-\cos x-\cos y-\cos z), \\
& U_{2}(x)=\frac{1}{2}(x+y+z-\cos x-\cos y-\cos z), U_{3}(x)=-\frac{1}{6}(x+y+z-\cos x-\cos y-\cos z), \\
& U_{4}(x)=\frac{1}{24}(x+y+z-\cos x-\cos y-\cos z), U_{5}(x)=-\frac{1}{120}(x+y+z-\cos x-\cos y-\cos z), \\
& U_{6}(x)=\frac{1}{720}(x+y+z-\cos x-\cos y-\cos z), \cdots
\end{aligned}
$$

Then, using the inverse transformation Eq.(2), we get the $n$th order approximate solution as,

$$
\tilde{u}(x, t)=\sum_{k=0}^{n} U_{k}(x) t^{k}=(x+y+z-\cos x-\cos y-\cos z)\left(1-t+\frac{1}{2 !} t^{2}-\frac{1}{3 !} t^{3}+\frac{1}{4 !} t^{4}-\frac{1}{5 !} t^{5}+\frac{1}{6 !} t^{6}+\cdots\right)
$$

which is the first eight terms of the poisson series of the exact solution Eq.(19).

Example 3.4 As the last example, consider the following three dimensional non-homogeneous fourth-order PDE [14]

$$
\frac{\partial^{2} u}{\partial t^{2}}+\frac{1}{4 ! z} \frac{\partial^{4} u}{\partial x^{4}}+\frac{1}{4 ! x} \frac{\partial^{4} u}{\partial y^{4}}+\frac{1}{4 ! y} \frac{\partial^{4} u}{\partial z^{4}}=\left\{-\frac{x}{y}-\frac{y}{z}-\frac{z}{x}+\frac{1}{x^{5}}+\frac{1}{y^{5}}+\frac{1}{z^{5}}\right\} \cos t, \frac{1}{2}<x, y, z<1, t>0
$$

subject to the initial condition 


$$
u(x, y, z, 0)=\frac{x}{y}+\frac{y}{z}+\frac{z}{x}, \frac{\partial u}{\partial t}(x, y, z, 0)=0
$$

whose exact solution is

$$
u(x, y, t)=\left(\frac{x}{y}+\frac{y}{z}+\frac{z}{x}\right) \cos t
$$

Applying RDTM to Eq.(23), the following recursive formula is obtained:

$$
\begin{aligned}
U_{k+2}(x, y, z)=\frac{-1}{(k+1)(k+2)} & \left\{\frac{1}{4 ! z} \frac{\partial^{4} U_{k}(x, y, z)}{\partial x^{4}}+\frac{1}{4 ! x} \frac{\partial^{4} U_{k}(x, y, z)}{\partial y^{4}}\right. \\
+ & \left.\frac{1}{4 ! y} \frac{\partial^{4} U_{k}(x, y, z)}{\partial z^{4}}-F_{k}(x, y, z)\right\}
\end{aligned}
$$

where $F_{k}(x)$ is the transformation of the function $f(x, y, z, t)=\left\{-\frac{x}{y}-\frac{y}{z}-\frac{z}{x}+\frac{1}{x^{5}}+\frac{1}{y^{5}}+\frac{1}{z^{5}}\right\} \cos t$.

From theorem 8, the redeuced differential transform $F_{k}(x)$ is,

$$
F_{k}(x, y, z)=\left\{-\frac{x}{y}-\frac{y}{z}-\frac{z}{x}+\frac{1}{x^{5}}+\frac{1}{y^{5}}+\frac{1}{z^{5}}\right\} \frac{1}{k !} \cos \left(\frac{k \pi}{2}\right)
$$

From Eq.(1), the initial conditions given in Eq.(24) can be transformed at $t=0$ as

$$
U_{0}(x, y, z)=\frac{x}{y}+\frac{y}{z}+\frac{z}{x}, U_{1}(x, y, z)=0
$$

Substituting Eq.(27) and Eq.(28) into Eq.(26) and by straightforward iterative steps, the following $U_{k}(x)$ (for $\left.\mathrm{k}=0,1,2, \ldots, \mathrm{n}\right)$ values are obtained.

$$
\begin{aligned}
& U_{0}(x)=\frac{x}{y}+\frac{y}{z}+\frac{z}{x}, U_{1}(x)=0, U_{2}(x)=-\frac{1}{2 !}\left(\frac{x}{y}+\frac{y}{z}+\frac{z}{x}\right), U_{3}(x)=0, \\
& U_{4}(x)=-\frac{1}{4 !}\left(\frac{x}{y}+\frac{y}{z}+\frac{z}{x}\right), U_{5}(x)=0, U_{6}(x)=\frac{1}{6 !}\left(\frac{x}{y}+\frac{y}{z}+\frac{z}{x}\right), U_{7}(x)=0, \cdots
\end{aligned}
$$

Then, using the inverse transformation Eq.(2), we get the $n$th order approximate solution as,

$$
\begin{aligned}
\tilde{u}(x, t)=\sum_{k=0}^{n} U_{k}(x) t^{k} & =\frac{x}{y}+\frac{y}{z}+\frac{z}{x}-\frac{1}{2 !}\left(\frac{x}{y}+\frac{y}{z}+\frac{z}{x}\right) t^{2}+\frac{1}{4 !}\left(\frac{x}{y}+\frac{y}{z}+\frac{z}{x}\right) t^{4}-\frac{1}{6 !}\left(\frac{x}{y}+\frac{y}{z}+\frac{z}{x}\right) t^{6}+\cdots \\
& =\left(\frac{x}{y}+\frac{y}{z}+\frac{z}{x}\right)\left(1-\frac{1}{2 !} t^{2}+\frac{1}{4 !} t^{4}-\frac{1}{6 !} t^{6}+\cdots\right)
\end{aligned}
$$

which is the first eight terms of the poisson series of the exact solution Eq.(25).

\section{Conclusion}


In this paper, we introduced the modified version of the DTM, namely the reduced differential transform method (RDTM) for solving fourth-order parabolic PDEs. The main advantage of the RDTM is to provide the user an analytical approximation to the solution, in many cases, an exact solution, in a rapidly convergent sequence with elegantly computed terms. The solution procedure of the RDTM is simpler and effective than other analytic methods such as the Adomian Decomposition Method (ADM), the Variational Iteration Method (VIM), the Homotopy Perturbation Method (HPM) and Spline methods. The results show that the RDTM is a powerful and effectiveness method for solving linear and nonlinear PDEs.

\section{References}

[1] A.Q.M. Khaliq and E.H. Twizell, A family of second-order methods for variable coefficient fourth-order parabolic partial differential equations, Int. J. Comput. Math., 23 (1987), 63-76.

[2] D.J. Gorman, Free Vibrations Analysis of Beams and Shafts, Wiley, New York ,1975.

[3] C. Andrade and S. McKee, High frequency A.D.I. methods for fourth-order parabolic equations with variable coefficients, Int. J. Comput. Appl. Math., 3 (1977), 11-14.

[4] S.D. Conte, A stable implicit difference approximation to a fourth-order parabolic equation, J. Assoc. Comp. Mach., 4 (1957), 210-212.

[5] S.D. Conte and W.C. Royster, Convergence of finite difference solution to a solution of the equation of a vibration beam, Proc. Amer. Math. Soc., 7 (1956), 742-749.

[6] D.J. Evans, A stable explicit method for the finite difference solution of fourth-order parabolic partial differential equations, Comp. J., 8 (1965), 280-287

[7] D.J. Evans and W.S. Yousef, A note on solving thefourth-order parabolic equation by a AGE method, Int. J. Comput. Math., 40 (1991), 93-97.

[8] K. Arshad, K. Islam and A. Tariq, Sextic spline solution for solving a fourth-order parabolic partial differential equation, Int. J. Comput. Math., 82 (2005), 871-879.

[9] B.K. Belinda, S. Oliver and W. Michael, A fourth-order parabolic equation modeling epitaxial thin film growth, J. Math. Anal. Appl., 286 (2003), 459-490.

[10] Y.L. You and M. Kaveh, Fourth-order partial differential equations for noise removal, IEEE Trans. Image Process., 9 (2000), 1723-1730.

[11] X.H. Tang and C.I. Christov, Non-linear waves of the steady natural convection in a vertical fluid layer: A numerical approach, Math. Comput. Simul., 74 (2007), 203-213.

[12] M. Khan, M.A. Gondal and S. Kumar, A new analytical approach to solve exponential stretching sheet problem in fluid mechanics by variational iterative Pade method, The Journal of Mathematics and Computer Science (JMCS), 3(2) (2011), 135 144.

[13] A.M. Wazwaz, Analytical treatment for variable coefficients fourth-order parabolic partial differential equations, Appl. Math. and Comput., 123 (2001), 219-227.

[14] A.M. Wazwaz, Exact solutions for variable coefficients fourth-order parabolic partial differential equations in higherdimensional spaces, Appl. Math. and Comput., 130 (2002), 415-424.

[15] M.A. Noor, K.I. Noor and S.T. Mohyud-Din, Modified variational iteration technique for solving singular fourth-order parabolic partial differential equations, Nonlinear Anal-Theor, 71(12) (2009),630-640.

[16] J. Biazar and H. Ghazvini, He's variational iteration method for fourth-order parabolic equations, Comput. \& Math. with Appl., 54(7-8) (2007), 1047-1054. 
[17] R.C. Mittal and R.K. Jain., B-splines methods with redefined basis functions for solving fourth order parabolic partial differential equations, Appl. Math. and Comput., 217(23) (2011), 9741-9755.

[18] H. Caglar and N. Caglar, Fifth-degree B-spline solution for a fourth-order parabolic partial differential equations, Appl. Math. and Comput., 201(1-2) (2008), 597-603.

[19] T. Aziz, A. Khan and J. Rashidinia, Spline methods for the solution of fourth-order parabolic partial differential equations, Appl. Math. and Comput.,167(1) (2005), 153-166.

[20] J. Biazar and H. Ghazvini, Convergence of the homotopy perturbation method for partial differential equations, Nonlinear Anal. Real World Appl. , 10(5) (2009), 2633-2640.

[21] M. Matinfar and M. Saeidy, Application of Homotopy analysis method to fourth-order parabolic partial differential equations, Appl. Appl. Math.: An Int. J. (AAM), 5(1) (2010), 70- 80.

[22] Y. Keskin and G. Oturanç, Reduced differential transform method for partial differential equations, Int J. Nonlinear Sci. Numer. Simul., 10(6) (2009),741-9.

[23] P.K. Gupta, Approximate analytical solutions of fractional Benney-Lin equation by reduced differential transform method and the homotopy perturbation method, Comput. Math. Appl., 61(9) (2011), 2829-2842.

[24] R. Abazari and M Abazari,Numerical simulation of generalized Hirota-Satsuma coupled KdV equation by RDTM and comparison with DTM, Commun. Nonlinear Sci. Numer. Simul., 17(2) (2012), 619-629.

[25] K. Yıldırım, B. İbiş and M. Bayram, New solutions of the non linear Fisher type equations by the reduced differential transform, Nonlinear Sci. Lett. A 3 (1) (2012), 29-36.

[26] A. Haghbin and S. Hesam, Reduced Differential Transform Method For Solving Seventh Order Sawada Kotera Equations, The Journal of Mathematics and Computer Science (JMCS), 5 (1) (2012),53-59. 